\title{
Fluidics: the answer to problems of handling hazardous fluids
}

\author{
V. Tesă̌ \\ Institute of Thermomechanics ASCR, Prague, Czech Republic
}

\begin{abstract}
Dangerous fluids must be kept within a containment. This may be a problem in emergency situations which almost inevitably happen from time to time in mechanical pumps and flow control valves due to material fatigue, seizing, or screws getting loose. During maintenance and repair the fluid may be exposed. A solution is offered by no-moving-parts maintenance-free fluidics. Three classes of devices are discussed: A) passive flow controllers, B) valves controlled by an external signal, C) fluidic pumps driven by alternating flow.

Keywords: hazardous fluids, fluidics, fluidic pumps, fluidic valves.
\end{abstract}

\section{Introduction: handling dangerous fluids}

The essential rule of handling dangerous liquids and gases - hot, poisonous, chemically aggressive, explosive, radioactive, or biologically pathogenic [1] - is keeping them inside a protective enclosure. Not always considered is the permanence of the containment in exceptional situations, such as during maintenance or repair, which is almost inevitable with classical mechanical pumps and flow control devices, operating either by motion or by deformation of their components. They are prone to failures due to material fatigue, worn seals, seizure damage, or bolts or screws becoming loose.

\section{Fluidic devices}

A solution offers no-moving-part fluidics, a technique of generating and controlling fluid flows by phenomena taking place inside cavities with solid walls. There is nothing that may seize, be worn, or break. As a result, fluidic pumps and valves need no maintenance and are extremely reliable for long time. 


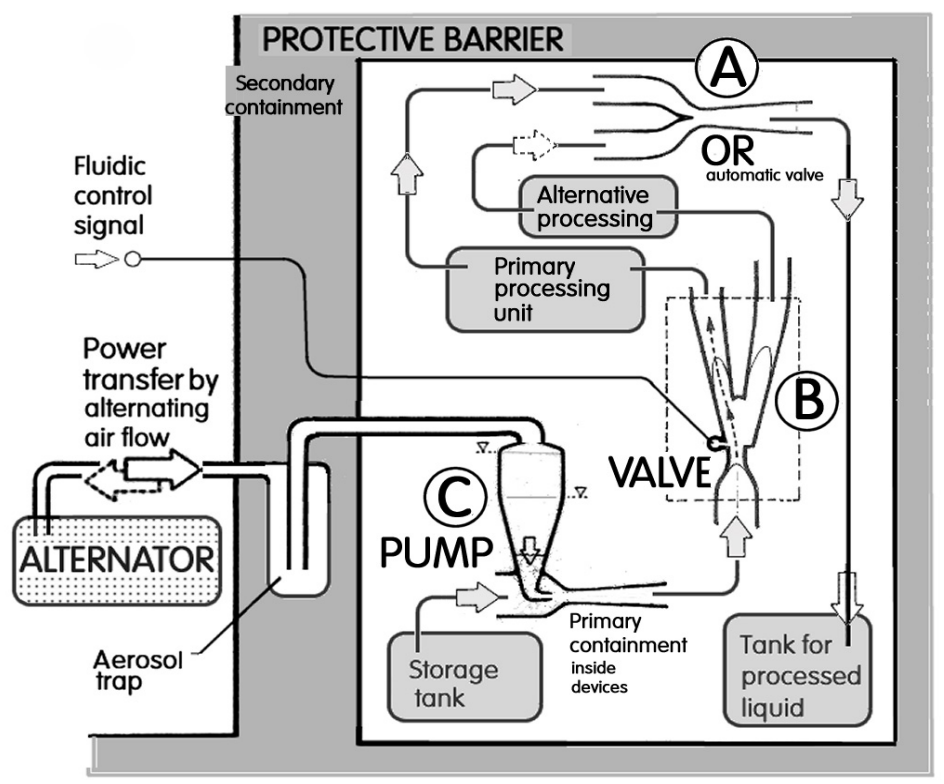

Figure 1: $\quad$ Schematic representation of a fluidic system handling a hazardous liquid inside a protective barrier. Three classes of no-moving-part devices are shown: $\mathrm{A}$ - passive flow control valves, B - valves controlled by small control flow from outside, $\mathrm{C}$ - pumps driven by alternating flow passing through the barrier.

Most engineers, if they have heard about fluidics at all, know it as the rather outdated technology that attempted in the 1970s, without success, to compete with electronics by processing signals carried by fluid flows. The absence of moving components enabled reaching operating frequencies higher than in classical hydraulics or pneumatics. Nevertheless, the limit of the speed of sound made it incomparably slower than electronics. Also the size of fluidic devices made them non competitive. Only recently a branch described as microfluidics [2] achieved small size, taking over the microfabrication methods.

The devices discussed here are different [3-7]. They are usually of large size [8], handling large flows. They are typically made without machining or assembly operations. This makes them quite inexpensive. Because of no need for an access for maintenance, the components and devices may be welded together to form a single, leak-proof solid body. They may be made of resistant, refractory materials, so that their operational life may be almost unlimited. Author's fluidic devices were originally developed for handling extremely dangerous radioactive fluids encountered in nuclear fuel re-processing. Nevertheless, their unique inherent safety properties make them the answer to many problems of transporting and processing other hazardous fluids.

If the handled fluid is really dangerous, several protective envelopes inside one another may be required. In the example in Fig. 1, in addition to the primary 
containment by the walls of the devices, there is the secondary outer containment of the whole fluid processing system inside the protection barrier. Typical requirements are: (a) absence of any mechanical moving devices beyond the barrier, and (b) adding there no (or minimum) supplementary fluid - to minimise the amount of what is to decontaminated or stored under special regime at the end of the processing. From this point of view, Fig. 1 classifies the devices into: A - fully passive, $B$ - requiring from outside just a very small control flow, C driven by an external flow. The requirement (a) above excludes power transfer across the barrier by an electric cable (since there is no way of moving fluid by electricity without a moving mechanical component) while (b) makes acceptable the class $\mathrm{C}$ only if the driving flow is alternating (sign changing).

In absence of moving components that would block the undesirable flowpath, the fluid in a fluidic device is forced to move into the desirable direction by its inertia. To make the inertial action effective, the fluid has to be accelerated usually in a nozzle. High flow speed, of course, means high hydraulic losses. To avoid them, the fluid is slowed down immediately down-stream from the interaction cavity by a diffuser, Fig. 2. Diffusers, as a result, are characteristic feature of most fluidic devices. Unfortunately, they are inconveniently long.

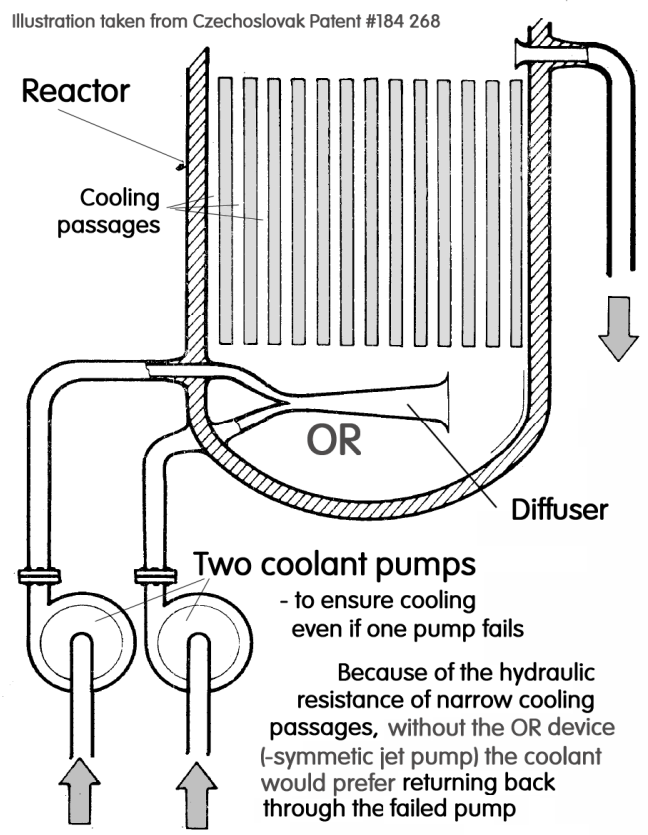

Figure 2: An example of class $A$ valve. Replacing mechanical check valves (which during long periods of no use may become immovable by rust) the failure-free, no-moving-part passive "logical OR" device [9] prevents any flow back through the failed pump. 


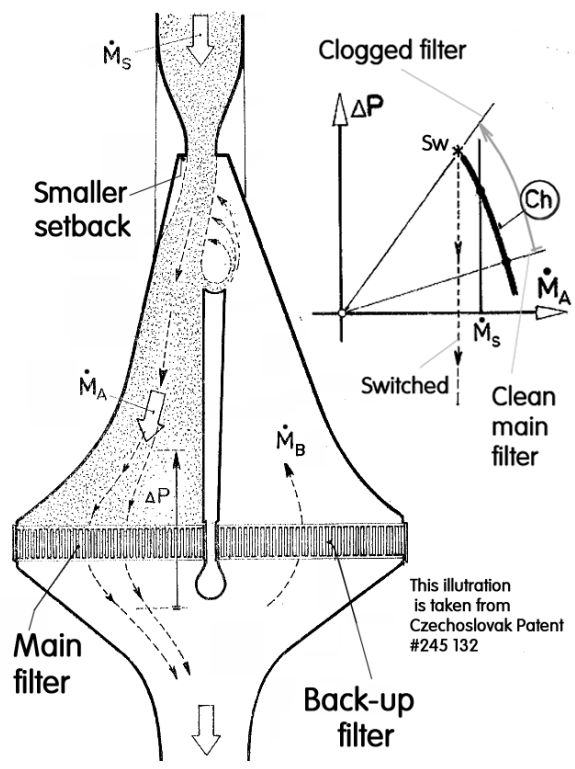

Figure 3: Another class $A$ valve. It switches the flow if the downstream resistance increases beyond the attachment capability of the Coanda effect. The original Patent [10], from which this picture is taken, concentrated on automatic insertion into the flowpath of a back-up filter once the main filter is clogged.

Also, improving their often low conversion efficiency is a perpetual challenge to the fluidic device designer. In fact, recent discovery [11] shows most of the conversion takes place prior to entry into the diffuser.

\section{Passive flow control valves: class $A$}

Examples are presented in Figs. 2-5. An interesting feature is the control in open loop, i.e. without feedback. Modern graphite-based materials make possible

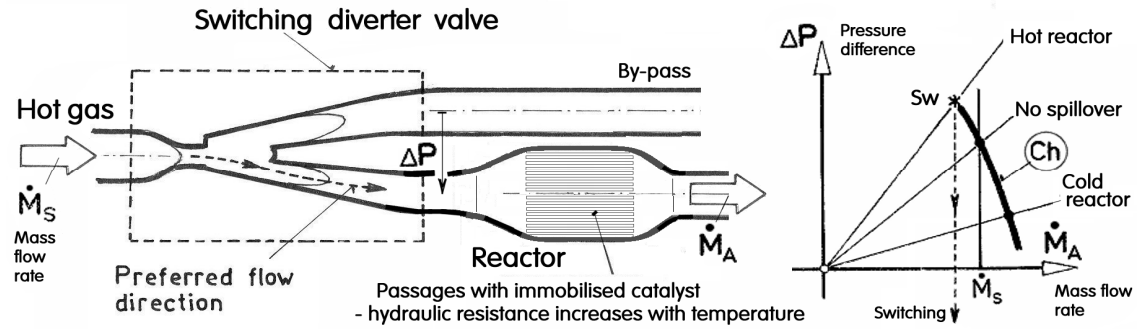

Figure 4: The same mechanism as in Fig. 3 applied to prevention of irreversible overheating of the reactor by the exothermic reaction (refs. [12-14]). 


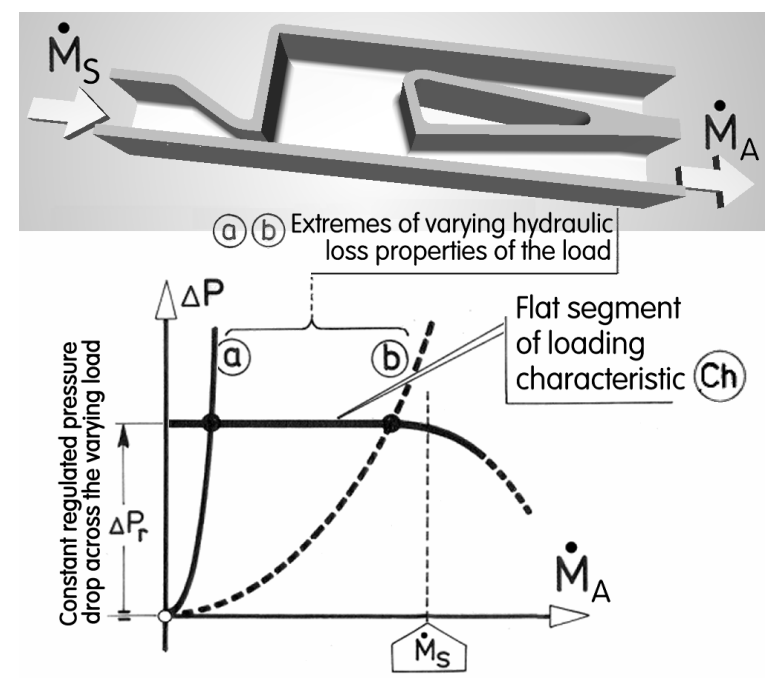

Figure 5: Pressure regulator class $A$ valve. The internal cavity is shaped to obtain the characteristic $\mathrm{Ch}$ with the flat part, ensuring at any loading constant output pressure (constant vertical position for intersection with characteristic within its segment $a$ to $b$ ). Details are in $[15,16]$.

manufacturing the devices for handling high melting point metals, such as molten steel [17]. The device shown in Figs. 1 and 2 prevents a reverse flow through one of the two parallel branches [8]. It is known (because of its role in control circuits of early days of fluidics) as the "logical OR". Essentially it is a symmetric (the same role of both inlets) jet pump.

More sophisticated are control valves employing special shape of their loading characteristic $\mathrm{Ch}$ - the dependence of the pressure drop $\Delta \mathrm{P}$ across the connected downstream load on the mass flow rate through it. The switching operation of the valves in Figs. 3 and 4 is based on the existence of the end of the curve Sw, beyond which the Coanda-effect no more keeps the jet at the preferential attachment wall [13] to which it initially attaches due to the smaller (Fig. 3) or no (Fig. 4) setback.

In another example, Fig. 5, described in [15], the valve geometry was developed so that the loading characteristic has the flat horizontal part. The valve operates as a pressure regulator and also load isolator (not reacting to the changes in the downstream connected load).

\section{Controlled valves: class B}

These devices, recently surveyed in [18], are essentially scaled up versions of the standard fluidic amplifiers [2]. The fluidic control signal may be derived from some change in the system inside the barrier, thus not breaking the rule (b). It may be, however, brought from outside because the amplifier property ensures 


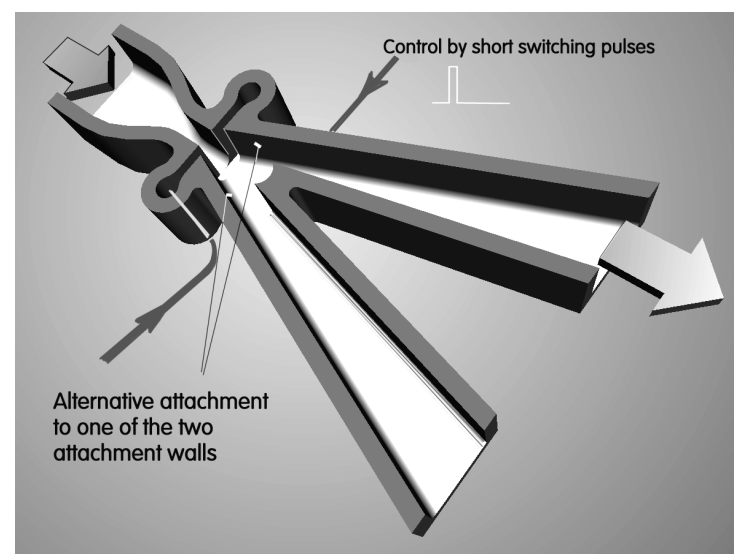

Figure 6: The advantage of Coanda-effect bistable diverter is the memory effect - the control of the switching is achieved by just a brief pulse.

the flow that crosses the barrier is relatively insignificant. This is particularly the case of the bistable valves of symmetric geometry (e.g., [19]) with equally feasible attachment by the Coanda-effect to one of the two walls, Fig. 6. The flow is switched by only brief pulses brought into the control nozzles.

The amplification property may be also used - by the addition of feedback loop channels from the output to input terminals - to convert the valve into an oscillator [19]. This is an extremely versatile device. In some processes in may be necessary to lead the fluid periodically into different processing units. The oscillation is also a way towards increasing heat and/or mass transfer by destroying the conduction layer held by viscosity on e.g. heat exchanger surface. Oscillators were also used in generation of fine droplets or fine bubbles [20].

\section{Fluidic pumps, class C}

Jet pumps - used by Philibert d'Orme as long ago as $1570(!)$ - are the oldest nomoving-part fluidic pumps. They are, unfortunately, breaking the rule (b) by adding the driving fluid to the processed one. This is avoided by transferring the power through the containment shell (Fig. 1) by an alternating flow. This may be generated by an external alternator, not working with the dangerous fluid. The conversion between the fluids, e.g. from air to the dangerous liquid, as well as the rectification is performed inside (there may be an aerosol trap somewhere near the barrier).

For the rectification without moving parts, considerable ingenuity was spent, with no particular success, on development of labyrinth-type fluidic diodes (the earliest: patent by N. Tesla, 1920). Also not suitable for the purpose discussed here are the simple Venturi type diodes, consisting of a nozzle followed immediately by a diffuser, despite their current popularity in microfluidics [2] where efficiency is not the primary factor while the high operating frequency 


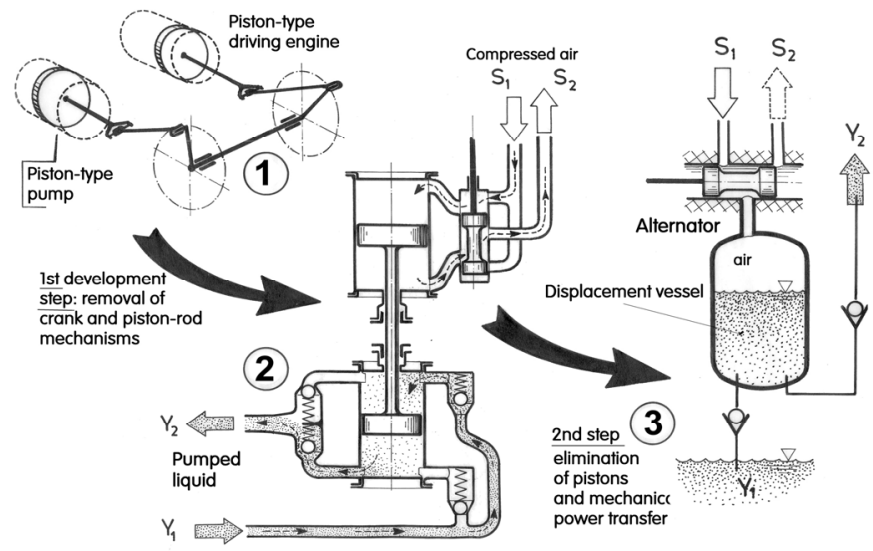

Figure 7: No-moving-part fluidic pump operate on the displacement principle: the alternating air flow that passes through the outer containment shell (Fig. 1) acts on the surface of the pumped dangerous liquid in the displacement vessel, not needing the separating piston.

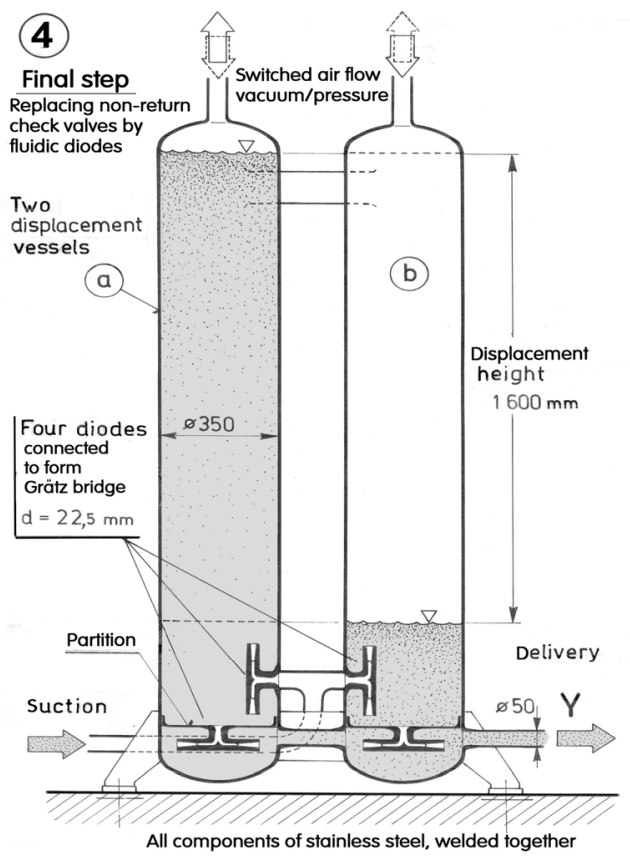

Figure 8: Author's two-phase pump with vortex diodes as an example of the slow type pump with the flow attaining almost steady state conditions. 

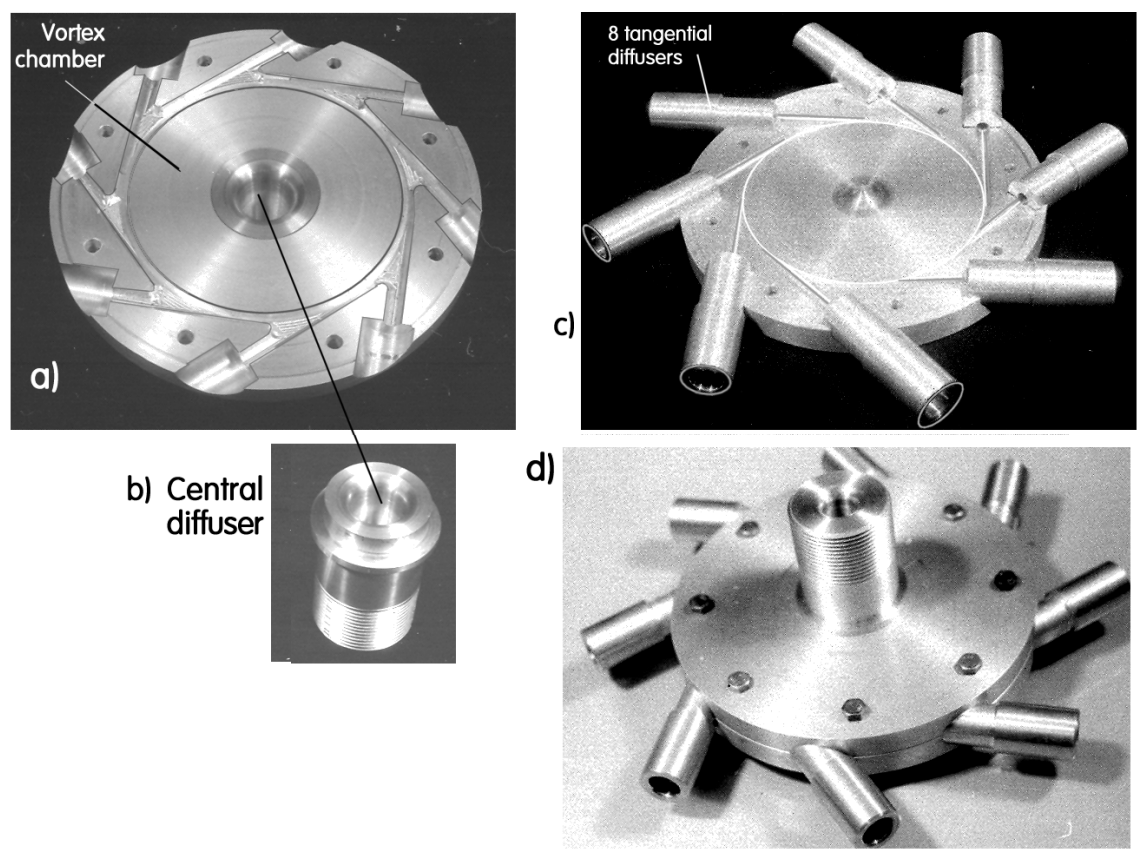

Figure 9: Photographs of the model diodes used in laboratory tests of the pump shown in Fig. 8. The large number of tangential inlets is necessary to ensure symmetry of the vortical flow. For other details consult ref. [7].

achievable is important. Really successful are the vortex diodes, Figs. 8-10. Those used in the author's pump shown in Fig. 8 exhibited 59-times higher pressure drop in the return flow direction than in the forward direction at the same flow rate $10 \times 10^{-3} \mathrm{~kg} / \mathrm{s}$. Details, including all dimensions, are in [7]. Their weakness is considerable time needed for rotation start-up. This is why the pumps like the one shown in Fig. 8 are operated with very long periods between switching (note the large volume of the displacement vessel).

\section{The FFD pump}

The original operating principle of the forward flow diverting was proposed already in 1976 [21, 22] for the rectifiers of high-frequency pumps. Essentially, its stage as presented in Fig. 11 is a Coanda-effect jet pump (with annular driving nozzle). In an attempt to increase the generated pressure difference, the pump was set up as tandem arrangement of several stages. The two-phase driving alternating flow was not rectified in the usual Grätz rectifying bridge, but led alternatively into the odd and even stages. Thus the generated pressure increases were summed. Also, this generated travelling waves, dragging the pumped fluid along - some of it not even entering the nozzles. A version of this novel pump, 

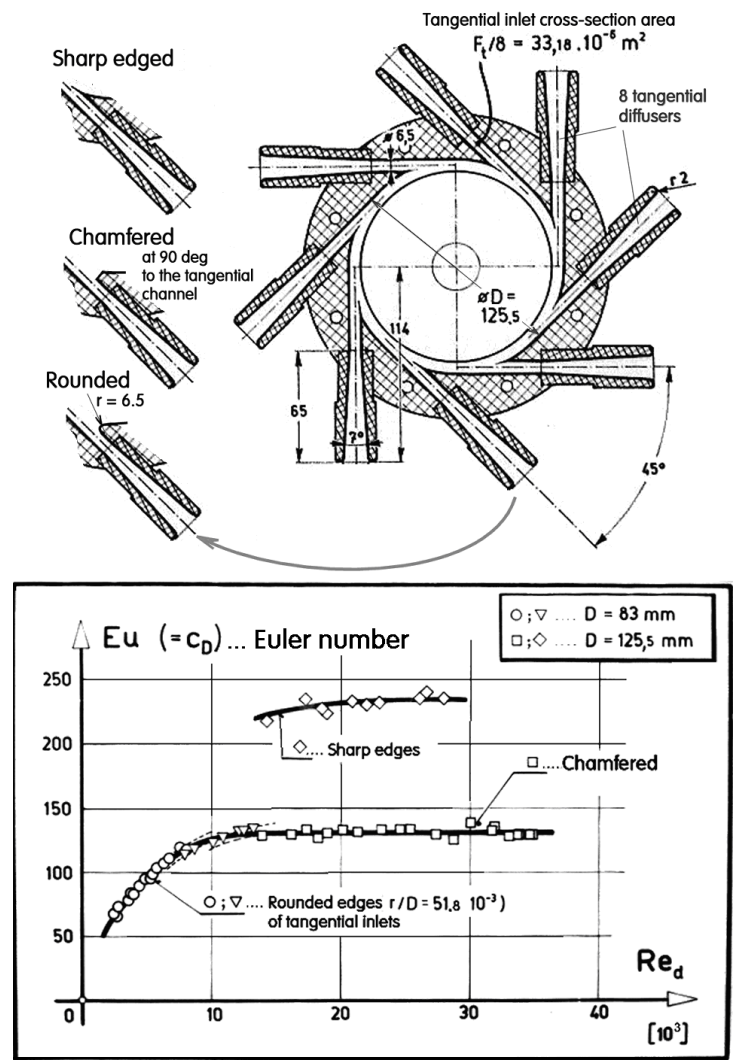

Figure 10: Vortex diode: both rounding or chamfering of the sharp edge at the entrance of the reverse ("OPEN" direction) flow into the tangetial diffusers were equally effective (the latter easier to do). Even more surprisingly [7], removal of this edge decreasd Eu also in the "CLOSED" direction, so that this expensive to make detail had small effect on the resultant rectification properties.

of $20 \mathrm{~mm}$ dia. bore, was recently tested as described in [6] in a project unfortunately terminated before several suggested improvements (described in [6]) could be tested. They were later incorporated in the small FFD pump shown here in Figs. 11-15. This pump re-circulating the sample in a preconcentrator for mass spectrometer analysis, an application also demanding an absolute leak-proof containment (here especially no contact with organic materials - sealants, lubricants). The laboratory model was test-driven by loudspeakers. The results in Fig. 14 show the improvement of properties with substantially increased cross-sectional area of the nozzle and the resonance at frequency as high as $\sim 190 \mathrm{~Hz}$. 


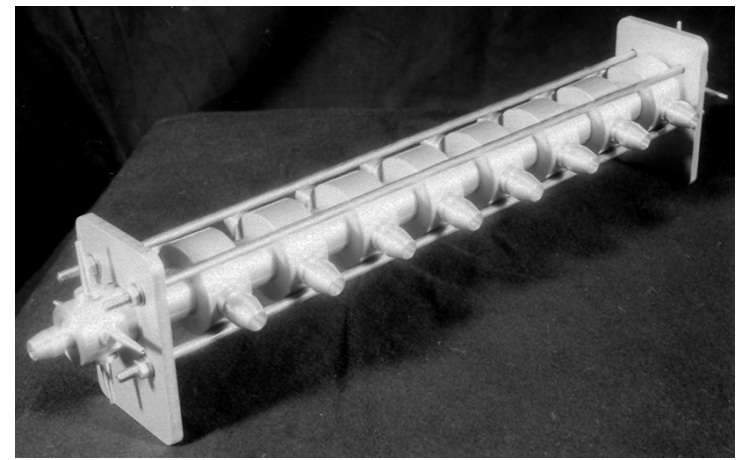

Figure 11: Assembled FFD pump model with 8 identical sections, each consisting of pair of the components $\mathrm{a}$ and $\mathrm{b}$ (Fig. 12).

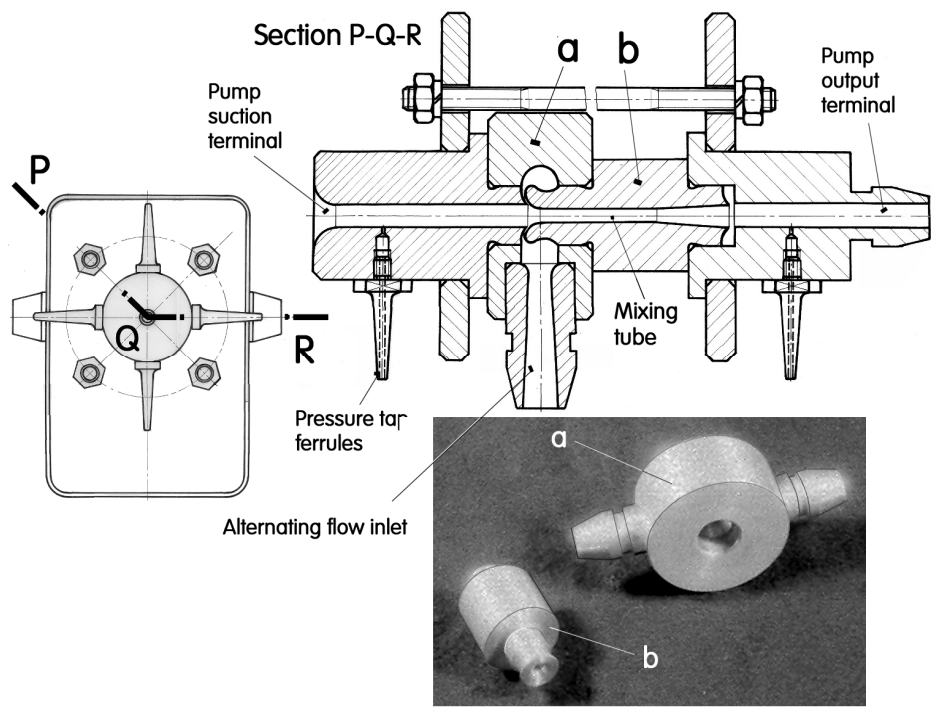

Figure 12: Basic components of the laboratory model of the high-frequency FFD pump. Only a single pair of components $a$ and $b$ is shown here, but actually used version had eight such pairs (Fig. 11) in series, alternatively driven by different phases of the alternating flows to generate travelling waves. 


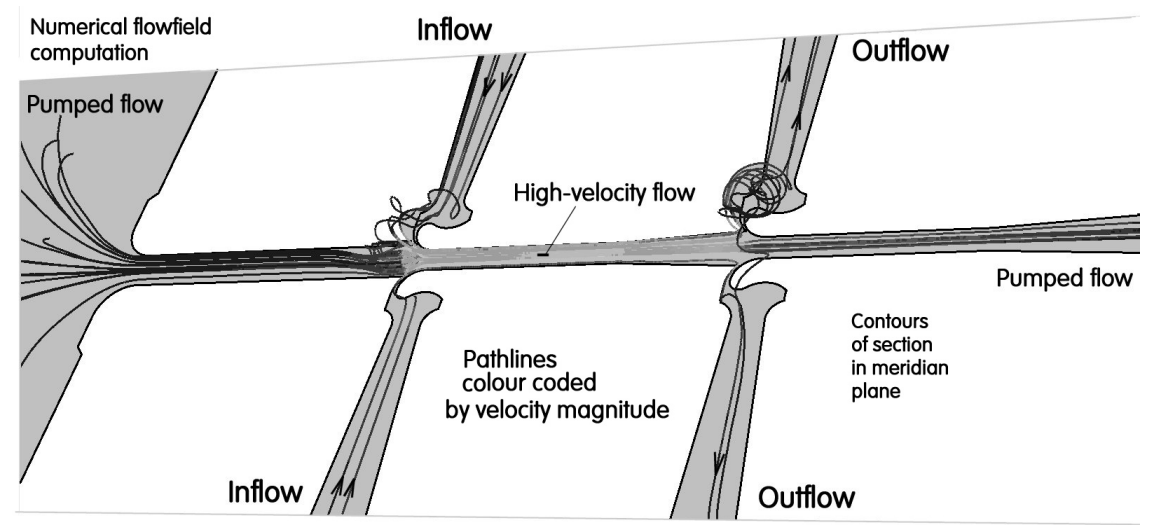

Figure 13: An example of numerical flowfield solutions: computed pathlines in the first two stages of the FFD pump from Figs. 11-15.

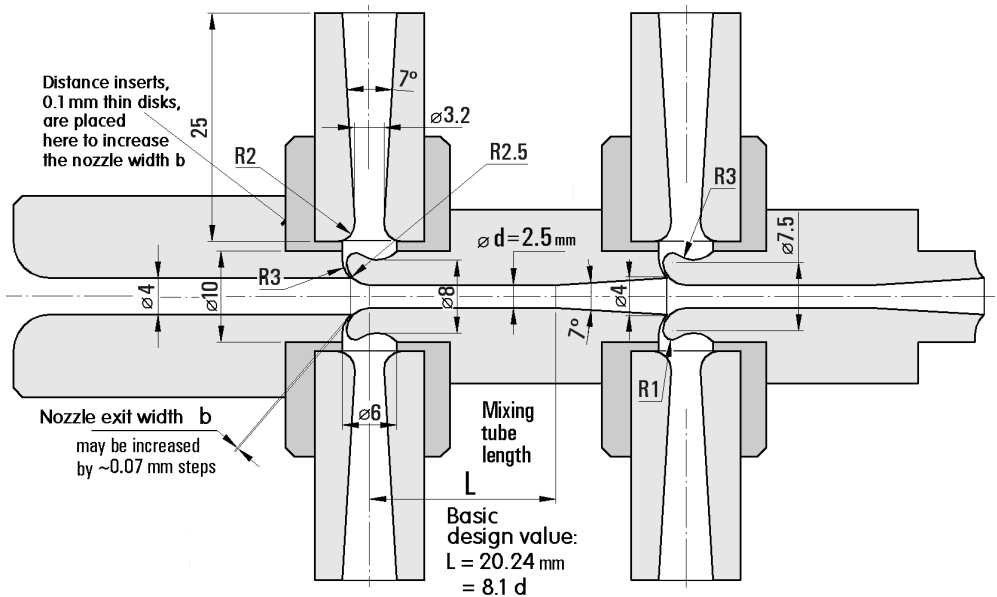

Figure 14: Geometry and dimensions of the basic configuration of the FFD pump (only two sections are shown). The length $L$ of the mixing tube and the nozzle exit widths $b$ were varied in the search for optimum. 


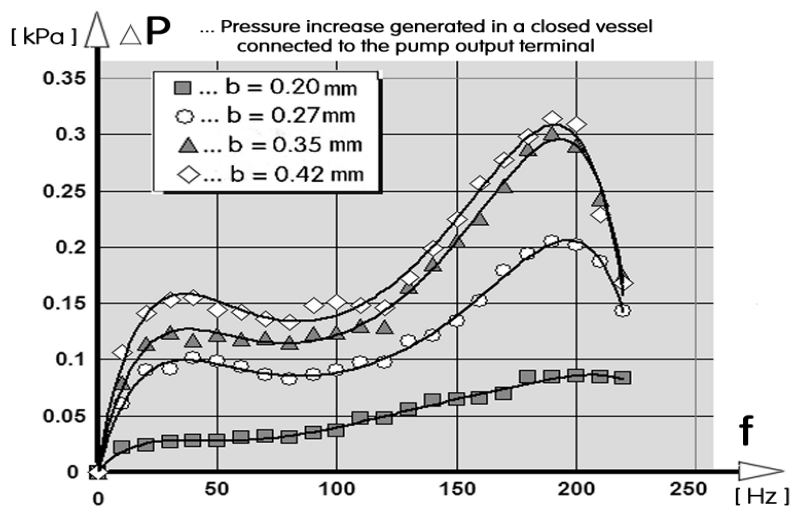

Figure 15 Experiments: the effect of the nozzle exit width $b$ on the pressure rise in a blocking, zero-flow-rate vessel connected to the output of the pump. The blockage is an adverse operating condition for this pump type.

\section{Conclusions}

The three described classes of no-moving-part devices [23] can generate and control hazardous fluid flows, ensuring permanent fluid containment. Their inherent safety and long-term reliability makes these little-known designs an ideal answer to many problems of dangerous fluids handling.

\section{Acknowledgements}

Gratefully acknowledged is the support by the research plan AV0Z20760514 and by the grant 101/11/J019 donated by GAČR

\section{References}

[1] The Stationery Office, Dangerous Goods Emergency. Action Code List, London, 2009

[2] Tesař V., Pressure-driven Microfluidics, Artech House Publishers, USA, 2007

[3] Tesař V., Little known principles of fluidics pumping, in: Proc. Int. Conf. "Experimental Fluid Mechanics 2010”, p. 716, Liberec, 2010

[4] Tesař V., Valve-less rectification pumps, in: D. Li (Ed.), Encyclopedia of Microfluidics and Nanofluidics, p. 2132, Springer, Wien, New York, 2008

[5] Tesař V., Configurations of fluidic actuators for generating hybrid-synthetic jets, Sensors and Actuators A: Phys. Vol. 138, p. 394, 2007

[6] Tesař V., Pump for Extremely Dangerous Liquids, Chemical Engineering Research and Design, 10.1016/j.cherd.2010.11.022, in press 
[7] Tesař V., Safe pumping of hazardous liquids - a survey of no-moving-part pump principles, Chem. Eng. J., DOI 10.1016/j.cej.2011.01.046, in press

[8] Tesař V.: Großmaßstäbliche fluidische Ventile für die Durchfluß-steuerung (Large-scale fluidic valves for flow control - in German), Messen-steuernregeln, Vol. 26, 1983

[9] Tesař V., Fluid exit into a vessel from two pipeline branches - in Czech), Czechoslovak Patent \# 184 268, Nov. 1976

[10] Tesař V., Filter for cleaning a fluid from carried particles - in Czech), Czechoslovak Patent \# 245 132, 1985

[11] Tesař V., Mechanism of Pressure Recovery in Jet-Type Actuators, Sensors and Actuators A-Physical, Vol. 152, p.182, 2009

[12] Tesař V., Fluidic Valves for Variable-Configuration Gas Treatment, Chemical Engineering Research and Design, Vol. 83 (A9), p. 1111, 2005

[13] Tesař V., No-Moving-Part Valve for Automatic Flow Switching, Chemical Engineering Journal, Vol. 162, p. 278, 2010

[14] Tesař V., Fluidic Valve for Reactor Regeneration Flow Switching”, Chemical Engineering Research and Design, Vol, 82 (A3), p. 1, 2004

[15] [15] Tesař V., Extremely Simple Pressure Regulator - Computational Studies, Chemical Engineering Journal, Vol. 155, p. 361, 2009

[16] Woods R.L., Tseng R.-J., A Fluidic Low Output Impedance Device, Proc. of FLUCOME '85, Fluid Control, Mechanics, Measurement, and Visualisation Symp., p. 229, Tokyo 1985

[17] Tesař V., Fluidic Control of Molten Metal Flow,_Acta Polytechnica - Journal of Advanced Engineering, Vol.43, p. 15, 2003

[18] Tesař V., Inherently safe diverter valves for flow control of hazardous fuids, in review, International Journal of Safety and Security Engineering

[19] Tesař V., Hung C.-H., Zimmerman W.: No-Moving-Part Hybrid-Synthetic Jet Actuator, Sensors and Actuators A, Vol. 125, p. 159, 2006

[20] Zimmerman W.B. et al., On the design and simulation of an airlift loop bioreactor with microbubble generation by fluidic oscillation, Food and Bioproducts Processing, Vol. 97, p. 215

[21] Tesař V., Čerpadlo nebo dmychadlo, zejména pro dopravu obtǐžně čerpatelných tekutin (Pump or blower, in particular for transporting fluids difficult to pump-in Czech), Czechoslovak Patent \# 1920 82, Apr. 1976.

[22] Tesař V., Active fluidic turn-down valve - in Czech, Czechoslovak Pat, \# 212802,1980

[23] Tesař V.: Valvole fluidiche senza parti mobili (Fluidic valves without moving parts - in Italian), Oleodinamica - pneumatica, Vol. 39, p. 216, 1998. 